Meta

Journal des traducteurs

Translators' Journal

\title{
Traduction et acceptabilité sociale : Cossery en version anglaise
}

\section{Françoise Miquet}

Volume 64, numéro 2, août 2019

URI : https://id.erudit.org/iderudit/1068198ar

DOI : https://doi.org/10.7202/1068198ar

Aller au sommaire du numéro

Éditeur(s)

Les Presses de l’Université de Montréal

ISSN

0026-0452 (imprimé)

1492-1421 (numérique)

Découvrir la revue

Citer cet article

Miquet, F. (2019). Traduction et acceptabilité sociale : Cossery en version anglaise. Meta, 64(2), 334-358. https://doi.org/10.7202/1068198ar
Résumé de l'article

Le regard cru, intemporel et dénué d'orientalisme que porte sur la société cairote l'écrivain égyptien d'expression française Albert Cossery, unique dans la littérature arabe, lui a valu une appréciation unanime en France. Or, la réception d'oeuvres de Cossery récemment publiées aux États-Unis révèle un écart culturel de part et d'autre de l'Atlantique, à l'égard de la critique comme de l'analyse littéraire : du côté anglo-saxon, on tend à envisager l'oeuvre sous une perspective postcoloniale et politiquement actuelle, alors que du côté français prévaut une approche davantage individuelle et historicisée, comme en témoigne l'intérêt que suscite en France la génétique littéraire. La traduction des passages susceptibles de heurter dans l'oeuvre cossérienne pose ainsi le problème de la fidélité à la voix de l'auteur, laquelle, décontextualisée et mise à distance de l'histoire personnelle de l'écrivain, résonne différemment. Les traductrices américaines, qui se sont dites sensibles à sa vision et touchées par ses personnages, ont néanmoins effectué dans ces passages problématiques des lissages à portée morale pour tenter de résoudre la tension entre adéquation au texte original et acceptabilité sociale de la traduction, telle qu'elle est définie par Gideon Toury (1995/2004).
Ce document est protégé par la loi sur le droit d'auteur. L’utilisation des services d’Érudit (y compris la reproduction) est assujettie à sa politique d'utilisation que vous pouvez consulter en ligne.

https://apropos.erudit.org/fr/usagers/politique-dutilisation/ 


\title{
Traduction et acceptabilité sociale: Cossery en version anglaise
}

\author{
FRANÇOISE MIQUET \\ Université de Montréal, Montréal, Canada \\ francoise.miquet@umontreal.ca
}

\section{RÉSUMÉ}

Le regard cru, intemporel et dénué d'orientalisme que porte sur la société cairote l'écrivain égyptien d'expression française Albert Cossery, unique dans la littérature arabe, lui a valu une appréciation unanime en France. Or, la réception d'œuvres de Cossery récemment publiées aux États-Unis révèle un écart culturel de part et d'autre de l'Atlantique, à l'égard de la critique comme de l'analyse littéraire: du côté anglo-saxon, on tend à envisager l'œuvre sous une perspective postcoloniale et politiquement actuelle, alors que du côté français prévaut une approche davantage individuelle et historicisée, comme en témoigne l'intérêt que suscite en France la génétique littéraire. La traduction des passages susceptibles de heurter dans l'œuvre cossérienne pose ainsi le problème de la fidélité à la voix de l'auteur, laquelle, décontextualisée et mise à distance de l'histoire personnelle de l'écrivain, résonne différemment. Les traductrices américaines, qui se sont dites sensibles à sa vision et touchées par ses personnages, ont néanmoins effectué dans ces passages problématiques des lissages à portée morale pour tenter de résoudre la tension entre adéquation au texte original et acceptabilité sociale de la traduction, telle qu'elle est définie par Gideon Toury (1995/2004).

\section{ABSTRACT}

The Egyptian author Albert Cossery, who wrote in French, provides a raw, timeless and yet devoid of orientalism view of Cairo society which is unique in Arab literature, and has garnered him unanimous appreciation in France. However, the reception of Cossery's recently published works in the United States reveals a transatlantic cultural gap, both in terms of criticism and literary analysis. While the Anglo-Saxon world tends to view the work from a postcolonial and politically contemporary perspective, on the French side a more individual and history-grounded approach prevails, as attested by the interest literary genetics arouses in France. The translation of some passages likely to shock in Cossery's novels thus raises the issue of fidelity to the author's voice, which resonates differently as it is decontextualized and distanced from Cossery's personal history. The American translators have displayed openness to the writer's vision and empathy with his characters; nevertheless, they have carried out some smoothing out of a moral nature in these problematic passages, possibly in an attempt to resolve the tension between the adequacy and social acceptability of the translation as defined by Gideon Toury (1995/2004).

\section{RESUMEN}

La visión cruda, atemporal y desprovista de orientalismo de la sociedad de El Cairo que el escritor egipcio francófono Albert Cossery propone de esta ciudad es única en la literatura árabe y le ha valido una apreciación unánime en Francia. Sin embargo, la recepción de las obras recientemente publicadas de este autor en Estados Unidos revela un vacío cultural a ambos lados del Atlántico, tanto en términos de crítica como de análisis literario: en el lado anglosajón, hay una tendencia a ver la obra desde una perspectiva postcolonial y políticamente contemporánea, mientras que en el lado francés prevalece un enfoque más individual e históricizado, como lo demuestra el interés que suscita la 
genética literaria en Francia. La traducción de los pasajes que pueden ser polémicos en la obra de Cossery plantea así el problema de la fidelidad a la voz del autor, la cual, descontextualizada y alejada de su historia personal, resuena de manera diferente. A pesar de que las traductoras estadounidenses afirmaron su sensibilidad a la visión del escritor y su empatía por sus personajes, utilizarón estrategias de nivelación de carácter moral en estos pasajes problemáticos, posiblemente con el fin de resolver la tensión entre la adecuación y la aceptabilidad social de la traducción tal como fue definida por Gideon Toury (1995/2004).

\section{MOTS CLÉS/KEYWORDS/PALABRAS CLAVE}

littérature égyptienne, traduction littéraire, postcolonialisme, censure, génétique littéraire Egyptian literature, literary translation, postcolonialism, censorship, literary genetics literatura egipcia, traducción literaria, post-colonialismo, censura, genética literaria

Les personnages sont là pour exprimer mes idées. J’aimerais qu'après avoir lu un de mes livres, les gens n'aillent pas travailler le lendemain, qu'ils comprennent que l'ambition de vivre est suffisante, que nulle autre ambition ne vaut!

(Cossery $\left.2005^{1}: 95\right)$

\section{Introduction}

Albert Cossery est un écrivain égyptien d'expression française dont la description crue et humoristique (cynique, selon certains) de ses concitoyens le classe à part dans la littérature arabe francophone. Cet auteur culte en France est peu connu aux ÉtatsUnis, où trois de ses œuvres ont récemment fait l'objet d'une traduction en anglais. Or, l'étude de deux de ces traductions et de la révision d'une traduction plus ancienne révèle de nombreux lissages et quelques omissions, dont l'une amène à s'interroger sur une censure potentielle.

Après avoir présenté l'auteur, son œuvre, la réception critique de celle-ci auprès des lectorats français et américain, ainsi que ses traductions anglaises, nous examinerons ces gestes traductifs qui visent à atténuer l'effet péjoratif de certains passages dans la traduction (Cossery 1955/1981) du roman Mendiants et orgueilleux (1955), dans la version révisée de cette traduction (Cossery 1955/2011) et dans la traduction (Cossery 1964/2010) de La violence et la dérision (1964). Puis, nous tenterons de les analyser à la lumière de la réception récente des œuvres de Cossery dans un monde littéraire anglo-saxon davantage influencé par une perspective postcoloniale, à la différence d'une réception française marquée par une approche littéraire plutôt individuelle combinée avec le statut d'auteur culte de Cossery. Enfin, nous recourrons à la tension entre adéquation au texte original et acceptabilité d'une traduction telle qu'elle est définie par Gideon Toury (1995/2004) pour proposer une interprétation de ces interventions.

\section{Albert Cossery et la réception de son œuvre en France}

\subsection{Un auteur égyptien qui écrit en français}

Né en 1913 dans une famille de propriétaires terriens d'Égypte, Albert Cossery fait ses études en français chez les frères catholiques, lisant Baudelaire, Zola et d'autres classiques. Ayant su très jeune qu'il deviendrait écrivain, il découvre Paris à 18 ans. 
Après des années dans le Vieux-Caire, où il fréquente autant les quartiers populaires que le mouvement surréaliste cairote, il s'intègre au Saint-Germain-des-Prés d'aprèsguerre et élit domicile dans un hôtel où il mènera une vie de dandy pauvre jusqu'à sa mort en 2008, à l'âge de 94 ans $^{2}$. Ses premiers essais littéraires, Les hommes oubliés de Dieu (1941) et La maison de la mort certaine (1944), impressionnent notamment Henry Miller, qui le fera connaître aux États-Unis. Cossery produira au total huit œuvres.

Le héros cossérien est un homme intelligent, charismatique, débrouillard, qui - parfois après une phase idéaliste ${ }^{3}$ - a appris à se réjouir du spectacle grotesque des puissants de ce monde. Cossery s'identifie à ce personnage et, selon ses propres dires, s'exprime par sa voix (Cossery, dans Cossery et Mitrani 1995: 93, 102; Armel 2008: par. 14). Le protagoniste principal est entouré d'autres hommes - marginaux provoquant l'autorité sous toutes ses formes, fonctionnaires en rupture de ban, mendiants philosophes ou voleurs poètes - qui, à des degrés divers, prennent part à cette comédie ou la contemplent.

Si les deux premiers ouvrages expriment des velléités de révolte sociale, c'est l'impossibilité de la révolution, présentée comme l'envers symétrique, tout aussi nocif, du pouvoir, qui s'affirme au fil d'œuvres réitérant avec une "remarquable cohérence» (Losfeld 2005: 5; Parris 2009: 135) que la seule posture possible face à l'absurdité de la condition humaine est la jouissance de la vie et la dérision. Cossery, qui n'est pas retourné en Égypte entre 1945 et 1975, a affirmé que «la ville a changé, c'est sûr, mais pas le petit peuple. Il a toujours cet humour qui défie la tyrannie imbécile du pouvoir et de la richesse» (Ayad 1999: par. 8).

Cossery se disait «écrivain égyptien qui écrit en français» (Bitton 1996: 64) «n'[ayant] jamais trahi [son] pays» (Cossery, dans Cossery et Mitrani 1995: 10) et affirmait que ses intrigues se déroulent dans une Égypte immuable tout en visant une dimension universelle. Comme d'autres écrivains égyptiens francophones, il a choisi l'exil définitif à Paris. Sa trajectoire est, selon l'arabisant et traductologue Richard Jacquemond (2000: 17), «la plus marquée par la spécificité égyptienne», et sa langue balzacienne "fait entendre l'arabe dans le français» (Jacquemond 1993: par. 38) autant par le rythme et l'abondance d'adjectifs que par certaines formulations (Jacquemond 1993, 2000), ce qu'affirment également d'autres auteurs (Fili-Tullon 2009; Fenoglio 1999; Khalifa 2012).

\subsection{Cossery vu par un traductologue arabisant}

Selon Jacquemond, qui s'intéresse à la littérature du monde arabe et notamment à celle de l'Égypte, Cossery «s'affranchit du naturalisme dominant» et «innove au moyen d'une politique de la démesure, de l'hyperbole et de la dérision qui casse l'“effet de réel”" (Jacquemond 2000: 17). Il s'agit d'un précurseur, voire d'un visionnaire:

Il n'est pas exagéré de dire que Cossery montre la voie, en français et vingt ans avant ses compatriotes de la "génération des années soixante», de ce que pourrait être un «réalisme magique», à la manière sud-américaine, dans la littérature arabe. Par son invention langagière, [...] il innove au sein même du modèle du roman européen en y intégrant une esthétique spécifique, issue de la littérature arabe. (Jacquemond 2000 : 18)

De manière générale, selon Jacquemond, la traduction des œuvres en langue arabe vers le français a souffert de 
l'oscillation entre orientalisation ou traduction exotisante, d'une part, et francisation, traduction naturalisante, transparente, de l'autre, [qui] est au principe de l'économie et de la poétique de la traduction française de la production arabe moderne. (Jacquemond 1993: par. 31)

En écrivant directement en français, Cossery aura, selon lui, échappé à la traduction orientalisante, assortie de l'appareil critique des spécialistes qui dominent le champ ${ }^{4}$ et "guident» le lecteur tout en «exotisant» l'original. Cossery lui-même souligne:

[les personnages] je les traduis de l'arabe. [...] Je pense en arabe, c'est-à-dire, je donne à ma phrase un tour qui n'est pas un tour parisien ou disons, occidental. Pour les conversations et les répliques, je pense en arabe. (Cossery, dans Cossery et Mitrani 1995: 90)

Cossery aura aussi échappé à la «traduction francisante» consistant à rendre le texte à la fois élégant et accessible au prix d'une certaine banalisation (Jacquemond 1993: par. 38).

Jacquemond avance par ailleurs que le «moment colonial» (Jacquemond 2000: 9) a vu fleurir en Égypte une élite locale, laquelle, se rapprochant des sphères du pouvoir, adopte les langues coloniales (le français, puis l'anglais), rendant moins pressante la nécessité de la traduction. Les langues étrangères permettent également d'échapper aux contraintes sociales de l'expression littéraire en arabe. "C'est l'“âge d'or" de la littérature d'expression française en Égypte» (Jacquemond 2000: 11), malgré la domination coloniale anglaise, justement parce que la France, n'étant pas la métropole coloniale, peut symboliquement «jouer le rôle de mère des arts et des lettres émancipatrice» (Jacquemond 2000: 14). Toujours selon Jacquemond:

Il est frappant de voir comment les principaux écrivains égyptiens semblent s'être approprié cette langue «naturellement», sans arrière-pensée, et l'on peut penser qu'ils étaient d'autant plus enclins à adhérer à l'idéologie universaliste française et à la reproduire qu'elle leur permettait de saper les bases de la domination anglaise. (Jacquemond 2000: 14)

Ainsi, si l'écrivain Cossery ne peut être abstrait du contexte colonial, sa position ne saurait être circonscrite à des paramètres de dominant/dominé.

\subsection{Un statut d'écrivain culte, une critique littéraire française peu abondante}

Longtemps restreinte à un cercle réduit d'amateurs, l'œuvre de Cossery atteint une certaine popularité dans les années 1990. Soudainement objet d'attention médiatique, il reçoit divers prix, voit ses œuvres complètes rééditées et inspire des créateurs de bandes dessinées $\left(G\right.$ olo ${ }^{5}$ ), de documentaires (voir annexe 3) et d'essais. Désormais auteur culte, il devient un personnage public qui se met en scène lui-même, dont les commentaires sont réifiés, et fait l'objet de commentaires respectueux, voire dithyrambiques. «Moraliste sans faille» (Leroy 2011: par. 2), «Voltaire du Nil» (Aïssaoui 2008; Ammoun 2008, titre), il revendique lui-même son aura orientale dans plusieurs entrevues. Si de rares commentateurs (Mitrani, dans Cossery et Mitrani 1995; Leroy 2011), par ailleurs proches de lui, osent interroger la place des femmes dans ses romans, la misogynie pourtant très visible dans son œuvre ne fait pas l'objet de cri- 
tiques. La seule voix discordante est celle d'Andrau (2013), qui aborde la personnalité de l'écrivain sous un angle plus critique. Christin (2017) s'adresse à Cossery à titre de maître à penser de l'affranchissement du matérialisme.

La vision de Cossery a pu être apparentée à l'anarchisme, voire à l'absurde et au nihilisme et ainsi dépolitisée (Jacquemond 2000: 17); d'aucuns insistent sur une incitation à la paresse qui serait instituée en philosophie de vie. Du côté des publications scientifiques en langue française, l'œuvre de Cossery est généralement envisagée dans une perspective littéraire et philologique y compris un angle social, voire sociologique, mais rarement postcolonial. Toutefois, selon Marc Kober, Cossery propose en fait une vision sociale:

Cossery rejoint [...] toute une série de romanciers réalistes et politiquement engagés qui dénoncent le scandale de la pauvreté des uns et de l'enrichissement des autres [...]. La vision du Caire chez Cossery est profondément sociale et politique. L'auteur [...] possède une vision globale de la répartition sociale inégalitaire du Caire dans son ensemble. (Kober 2016: par. 13)

Les critiques littéraires de Cossery, peu nombreuses, abordent son œuvre sous l'angle de la peinture sociale, dans la tradition des classiques qui l'ont formé. Son «égyptianité» (Fenoglio 1999: 127-130), sa conception d'une société "où la fraternité a une part essentielle» (Gazio 1999: 110), son «savoir-vivre égyptien» (Gazio 1999: 105) et sa capacité à évoquer la torpeur et de la misère du Caire en créant une «émotion et une souffrance qui sont celles du témoin» (Kober 1999: 125) sont autant d'éléments soulignés par divers commentateurs. Parmi les textes scientifiques recensés, un seul aborde la place de la femme, mais élude la question de la misogynie en évoquant un trait culturel:

[...] l'une des marques [de cet humour] est le machisme, somme toute bon enfant, que l'on serait tenté de qualifier d'«oriental». Ainsi est-il souvent reproché aux femmes d'être insensibles à l'humour et d'être incapables d'ironie [...]. (Fili-Tullon 2009: 239; nos italiques)

\section{Les traductions anglaises des romans de Cossery et leur réception aux États-Unis}

\subsection{Plusieurs épisodes de traduction, un lectorat restreint}

Cossery n'a pas vu ses œuvres traduites en anglais à l'époque de leur publication: après Les hommes oubliés de Dieu (1941), La maison de la mort certaine (1944) et Les fainéants dans la vallée fertile (1948), respectivement parus en anglais sous les titres Men God Forgot (Cossery 1941/1946), The House of a Certain Death (Cossery 1944/1949) et The Lazy Ones (Cossery 1948/1952), près de trente années précéderont la traduction de Mendiants et orgueilleux (1955), Proud Beggars (Cossery 1955/1981). Il faudra encore trente autres années avant qu'en 2010 et 2011, peu après le décès de Cossery (2008), trois œuvres soient pour la première fois publiées en anglais ${ }^{6}$ : The Jokers (Cossery 1964/2010), traduction de La violence et la dérision (1964); A Splendid Conspiracy (Cossery 1975/2010), traduction de Un complot de saltimbanques (1975); et The Colors of Infamy (Cossery 1999/2011), traduction de Les couleurs de l'infamie (1999). Une révision de la première traduction de Mendiants et orgueilleux (1955) est effectuée dans la foulée (Cossery 1955/2011). 
Les maisons d'édition de The Jokers (Cossery 1964/2010) et The Colors of Infamy (Cossery 1999/2011), respectivement New York Review of Books (coll. Classics) et New Directions Books, s'adressent à un public au niveau d'éducation élevé, notamment susceptible d'empathie envers le sort des femmes et du «peuple» dans les sociétés arabes. Or, l'examen d'œuvres cossériennes écrites à différentes époques confirme que ces deux thématiques sont caractérisées par l'utilisation de termes et d'expressions connotés négativement ou véhiculant des idéologèmes ${ }^{7}$ (Angenot 1977: 24) susceptibles de heurter les sensibilités de ce lectorat.

\subsection{Représentations susceptibles de heurter le lectorat américain}

\subsubsection{Cossery et «la femme»}

Les femmes, chez Cossery, sont visibles essentiellement sous trois formes. D'abord la jeune fille, à qui son statut social «transitoire» permet de vivre avec le héros des relations sexuelles clandestines non dénuées de sentiments, mais toujours vouées à prendre fin lorsque la protagoniste devient femme et perd une intelligence, voire une mystérieuse et vive compréhension du monde inhérente à cet archétype cossérien. Selon Fili-Tullon (2009: 238), «la jeunesse selon la vision cossérienne est un état de grâce menacé par l'âge adulte». La prostituée est souvent une très jeune fille qui joue de séduction et de manipulation sans toutefois prétendre au niveau d'intelligence du personnage masculin, à qui elle inspire de l'indifférence, plus rarement de la compassion. Enfin, la femme adulte n'apparaît que de façon anonyme, le plus souvent comme membre d'un groupe décrit comme étant stupide, criard et vulgaire. Essentiellement préoccupée par les biens de ce monde, elle ne se soucie nullement de réfléchir - à l'image de son mari, petit fonctionnaire conventionnel, lâche et limité intellectuellement.

Certes, il est légitime de se demander si une telle réduction ne s'inscrirait pas dans la vision d'une société telle que l'auteur la découvre dans les années 1930, puis la transporte avec lui, inchangée à jamais, doublée d'une forme de compassion ironique devant le destin étriqué auquel étaient vouées la grande majorité des femmes dans l'Égypte de l'époque (comme semble le suggérer Espinose 2009: 15). Un extrait du roman Une ambition dans le désert (1984) pourrait, peut-être, fournir un indice dans ce sens: "Il n'aimait que les très jeunes filles, d'abord par inclination - les femmes l'ennuyaient, car déjà imprégnées de sottise masculine [...]» (Cossery 1984/2005: 354; nos italiques) ${ }^{8}$. Cependant, malgré les trois décennies qui séparent l'extrait de Mendiants et orgueilleux (Cossery 1955: 105), dans lequel Gohar affirme: "Il est simplement sous l'influence de toute une littérature européenne qui prétend faire de la femme un mystère. El Kordi s'ingénie à croire que la femme est un être pensant [...]», et celui ci-dessous, des Couleurs de l'infamie (1999), la position de l'auteur, communiquée par le héros du roman, n'a pas changé:

1) Ce qu'il n'aurait jamais pu supporter d'une femme adulte, il l'acceptait volontiers de la jeune fille qui expérimentait à ses dépens la déraison et les ambiguïtés que d'éminents psychologues attribuent au mystère féminin. Comme Osama n'avait jamais décelé en aucune femme le moindre mystère, les artifices de Safira ne provoquaient habituellement en lui aucune perplexité, mais une vague pitié à l'égard de la bêtise universelle. (Cossery 1999/2000: 30-31; nous soulignons) 
Ainsi, il paraît difficile, selon les critères communément acceptés aujourd'hui, de ne pas voir dans l'œuvre cossérienne une misogynie se manifestant de façon parfois frappante, notamment dans cette métaphore comparant des promeneuses à des «vaches» regroupées «sous l'œil de leur propriétaire» (Cossery 1964/2005: 288), ou encore le vocable récurrent de «femelles». Il est vraisemblable que cette posture ait compliqué le positionnement des traductrices par rapport à l'auteur.

\subsubsection{Cossery et «le peuple»}

Certains critiques soulignent le pessimisme, voire le cynisme de la vision qu'a Cossery de l'humanité. D’autres s'émerveillent de sa capacité à dépeindre la misère la plus noire; Henry Miller aurait comparé l'auteur à Dostoïevski (Leroy 2011 : par. 17). La peinture que fait du "peuple» cet auteur, situant ses personnages et ses intrigues dans un décor qui se veut universel, est dénuée de tout romantisme et de tout exotisme:

Son plus grand mérite est d'avoir surmonté la tentation du pittoresque et de nous avoir épargné les inévitables scènes folkloriques auxquelles croient devoir sacrifier les écrivains qui traitent de l'Orient. (Henein 1956: 137, cité dans Jacquemond 2000: 18)

Par ailleurs, si complaisance il y a pour décrire les habitants de la "ville indigène» (Cossery 1955: 160), c'est dans le luxe d'adjectifs et de métaphores qui présentent un monde grouillant, insalubre, sauvage et violent bien que rempli d'humour, où les gens sont «implacablement animés d'une saine confiance en la vie»(Cossery 1955: 101). Gohar, l'ancien professeur d'université devenu clochard de Mendiants et orgueilleux (1955), en éprouve une fascination existentielle:

2) L'univers n'était pas absurde, il était seulement régi par la plus abominable bande de gredins qui eût jamais souillé le sol de la planète. [...] Le pouvoir sanguinaire n'avait aucune prise sur les individus qui ne lisaient pas les journaux. L'angoisse ne pouvait pas atteindre ces gens-là. La ville indigène se trouvait être par miracle le seul endroit du pays non encore violenté, et où s'épanouissait une vie saine, animée par la simple raison. Partout ailleurs, c'était le règne de la plus incroyable folie. (Cossery 1955: 159-160)

Les commentaires des critiques français et ceux de l'auteur lui-même soulignent l'amour du peuple à qui Cossery prête collectivement des vertus rédemptrices tout en dépeignant de façon crue son mode de vie et les rapports entre individus souvent impitoyables. Cette posture en apparence contradictoire, qui a pu laisser perplexe une partie de la critique américaine, aura sans doute également posé certains problèmes aux traductrices.

\subsection{Une réception critique américaine partagée}

Les traductions effectuées entre 2009 et 2011, combinées avec l'effet médiatique du Printemps arabe de 2011, ont fait redécouvrir Cossery outre-Atlantique. Contrairement à la presse française, la presse américaine se montre critique, notamment, de la misogynie de l'auteur et de son absence d'analyse politico-sociale:

More disturbing is the unmistakable tint of misogyny running throughout these novels, an old-school male chauvinism that neither Cossery's times nor his culture can excuse. [...] his novels, by offering few shades of gray, lack the depth that could have 
allowed them to be the powerfully ironic statements that some critics have taken them for. (Polizotti 2012: par. 16-17)

De toute évidence, Mark Polizotti, éditeur auprès du Metropolitan Museum of Art de New York, qui a traduit Duras et Flaubert, n'adhère assurément pas à l'ethos de Cossery. Un commentaire du New Inquiry se montre critique de la langue (traduite) de l'écrivain:

Throughout the novel, language devolves into puerile absurdity, creating Beckettian scenes counterpoising sense against non-sense. In the introduction to Proud Beggars, translator Alyson Waters explains that Cossery tried to mimic the translation of Arabic into French thus creating dissonance in the dialogue, broken by idiomatic expressions and entreaties to Allah. The result can be jarring, perhaps doubly so in English: bits of dialogue clashing with adjective laden-descriptions as we travel in and out of various heads, navigating the chaotic jumble of thoughts and impressions. (McCanne 2012: par. 9)

Bien entendu, l'on trouve aussi des commentaires positifs dans la critique américaine ${ }^{9}$. Toutefois, on est loin de l'unanimité souvent révérencieuse constatée en France.

\section{Interventions traductives de lissage et omission significative dans deux traductions récentes et la révision d'une traduction plus ancienne}

\subsection{Proud Beggars : révision par Alyson Waters de la traduction de T. W. Cushing}

Considéré par plusieurs comme le chef-d'œuvre de Cossery, Mendiants et orgueilleux est à la fois un roman policier et une fable morale dont l'antihéros, le policier homosexuel Nour El Dine, finira par adopter le mode de vie du criminel, Gohar, lui-même ancien professeur d'université devenu mendiant. Selon Waters (2011: xv), sa révision de la traduction de Thomas W. Cushing, qu'elle qualifie de "fine work», avait pour but de «faire en sorte que le lexique soit similaire dans les trois livres» et de «conserver la singularité du style de Cossery $»^{10}$. En effet, nous avons noté un certain nombre de changements stylistiques. Toutefois, une comparaison systématique entre l'original, la version de Cushing et la révision de Waters montre que la grande majorité des manipulations (traduction ou révision) ayant porté sur les passages susceptibles de choquer le lectorat cible ont eu pour effet d'en réduire la force expressive négative ou péjorative, ou d'en atténuer l'effet potentiellement choquant.

TABLEAU 1

Gestes traductifs d'atténuation observés dans la traduction anglaise de Mendiants et orgueilleux ainsi que dans la révision de cette traduction

\begin{tabular}{|l|l|l|l|}
\hline \multicolumn{1}{|c|}{$\begin{array}{c}\text { Mendiants et } \\
\text { orgueilleux } \\
\text { (Cossery 1955) }\end{array}$} & $\begin{array}{c}\text { Proud Beggars (Cossery } \\
\text { 1955/1981), traduction } \\
\text { de T. W. Cushing }\end{array}$ & $\begin{array}{c}\text { Proud Beggars (Cossery } \\
\text { 1955/2011), révision } \\
\text { d'Alyson Waters }\end{array}$ & $\begin{array}{c}\text { Effet de la traduction } \\
\text { et de la révision }\end{array}$ \\
\hline $\begin{array}{l}\text { La furtive vision d'un } \\
\text { tas de grosses femelles } \\
\text { vêtues d'amples mélayas } \\
\text { noires [...]. (p. 10) }\end{array}$ & $\begin{array}{l}\text { The furtive glimpse of a } \\
\text { pack of giant women } \\
\text { dressed in full black } \\
\text { melayas [...]. (p. 10) }\end{array}$ & $\begin{array}{l}\text { The fleeting vision of a } \\
\text { pack of giant women } \\
\text { dressed in full black } \\
\text { melayas [...]. (p. 6) }\end{array}$ & $\begin{array}{l}\text { Double perte de } \\
\text { connotation négative } \\
\text { dans les deux cas. }\end{array}$ \\
\hline
\end{tabular}




\begin{tabular}{|c|c|c|c|}
\hline $\begin{array}{l}\text { [...] le sol était jonché } \\
\text { d'enfants en bas âge, } \\
\text { aux yeux glaireux livrés } \\
\text { aux mouches, } \\
\text { semblables à de petites } \\
\text { bêtes rampantes. (p. 25) }\end{array}$ & $\begin{array}{l}{[\ldots] \text { the ground was }} \\
\text { strewn with young } \\
\text { children with glairy, } \\
\text { fly-covered eyes who } \\
\text { resembled little } \\
\text { crawling animals. } \\
\text { (p. 21) }\end{array}$ & $\begin{array}{l}{[\ldots] \text { the ground was }} \\
\text { strewn with young } \\
\text { children with glairy, } \\
\text { fly-covered eyes who } \\
\text { resembled little } \\
\text { crawling animals. } \\
\text { (p. 16) }\end{array}$ & $\begin{array}{l}\text { Légère perte de } \\
\text { connotation négative } \\
\text { dans les deux cas, } \\
\text { «livrés aux mouches» } \\
\text { étant plus pathétique } \\
\text { que «couverts de } \\
\text { mouches». }\end{array}$ \\
\hline $\begin{array}{l}\text { Dans cette pénombre } \\
\text { aux effluves de stupre } \\
\text { récent }[\ldots] \text { (p. } 36 \text { ) }\end{array}$ & $\begin{array}{l}\text { In this shadow scented } \\
\text { with recent debauches } \\
{[\ldots] \text { (p. 28) }}\end{array}$ & $\begin{array}{l}\text { In this half-light scented } \\
\text { with recent wantonness } \\
{[\ldots] \text { (p. 22) }}\end{array}$ & $\begin{array}{l}\text { Perte de force } \\
\text { péjorative dans les } \\
\text { deux cas, plus grande } \\
\text { dans la révision de } \\
\text { Waters. }\end{array}$ \\
\hline $\begin{array}{l}\text { C'était lui qui mariait } \\
\text { - affreux simulacre } \\
\text { - les détenus entre eux. } \\
\text { (p. 47) }\end{array}$ & $\begin{array}{l}\text { It was he who married } \\
\text { - frightful sham - the } \\
\text { convict couples. (p. } 37 \text { ) }\end{array}$ & $\begin{array}{l}\text { He was the one who } \\
\text { "married" - what a } \\
\text { mockery! - the convict } \\
\text { couples. (p. } 31 \text { ) }\end{array}$ & $\begin{array}{l}\text { Perte de force } \\
\text { péjorative dans la } \\
\text { révision de Waters. }\end{array}$ \\
\hline $\begin{array}{l}{[\ldots] \text { le médecin légiste }} \\
\text { examinait encore le } \\
\text { cadavre de la putain } \\
\text { assassinée }[\ldots] \text {... (p. } 68 \text { ) } \\
\text { (5 autres occurrences) }\end{array}$ & $\begin{array}{l}{[\ldots] \text { the coroner was }} \\
\text { still examining the } \\
\text { corpse of the murdered } \\
\text { prostitute. (p. 53) }\end{array}$ & $\begin{array}{l}{[\ldots] \text { the coroner was }} \\
\text { still examining the } \\
\text { corpse of the murdered } \\
\text { prostitute. (p. } 45 \text { ) }\end{array}$ & $\begin{array}{l}\text { Perte de force } \\
\text { péjorative dans les } \\
\text { deux cas, pour six } \\
\text { occurrences du mot } \\
\text { "putain " présentes } \\
\text { dans la narration. }{ }^{11}\end{array}$ \\
\hline $\begin{array}{l}\text { [les prostituées] } \\
\text { c'étaient des bêtes de } \\
\text { somme, stupides et } \\
\text { illettrées [...]. (p. } 73 \text { ) }\end{array}$ & $\begin{array}{l}{[\ldots] \text { they were all stupid, }} \\
\text { illiterate drudges }[\ldots] . \\
\text { (p. 57) }\end{array}$ & $\begin{array}{l}{[\ldots] \text { they were all stupid, }} \\
\text { illiterate drudges }[\ldots] \text {. } \\
\text { (p. 48) }\end{array}$ & $\begin{array}{l}\text { Perte de force } \\
\text { péjorative dans les } \\
\text { deux cas. }\end{array}$ \\
\hline $\begin{array}{l}\text { Certes, la misère } \\
\text { marquait leurs } \\
\text { vêtements composés de } \\
\text { hardes innommables } \\
{[\ldots] \text { (p. 93) }}\end{array}$ & $\begin{array}{l}\text { True, poverty marked } \\
\text { their clothes made of } \\
\text { innumerable rags }[\ldots] \text {. } \\
\text { (p. } 71)\end{array}$ & $\begin{array}{l}\text { True, poverty marked } \\
\text { their clothes made of } \\
\text { innumerable rags }[\ldots] . \\
\text { (p. 62) }\end{array}$ & $\begin{array}{l}\text { Erreur de traduction, } \\
\text { reproduite dans la } \\
\text { version révisée, qui } \\
\text { produit un effet } \\
\text { d'atténuation. }\end{array}$ \\
\hline $\begin{array}{l}\text { Tiens, maudit! Tu ne } \\
\text { veux pas m'enculer } \\
\text { encore! (p. 97) }\end{array}$ & $\begin{array}{l}\text { Here, you wretch! You } \\
\text { would be up my ass } \\
\text { anymore! (p. 74) }\end{array}$ & $\begin{array}{l}\text { Here, you wretch! You } \\
\text { would be up my ass } \\
\text { anymore! (p. 65) }\end{array}$ & $\begin{array}{l}\text { Glissement de sens et } \\
\text { perte de force } \\
\text { vulgaire dans les } \\
\text { deux cas. }\end{array}$ \\
\hline $\begin{array}{l}\text { De sa place, il } \\
\text { contemplait } \\
\text { distraitement ses affreux } \\
\text { collègues }[\ldots] .(\text { p. 113) }\end{array}$ & $\begin{array}{l}\text { From his chair, he } \\
\text { distractedly } \\
\text { contemplated his sorry } \\
\text { colleagues }[\ldots] . \text { (p. } 87 \text { ) }\end{array}$ & $\begin{array}{l}\text { From his chair, he } \\
\text { distractedly } \\
\text { contemplated his sorry } \\
\text { colleagues }[\ldots] . \text { (p. } 76 \text { ) }\end{array}$ & $\begin{array}{l}\text { Perte de force } \\
\text { péjorative dans les } \\
\text { deux cas. }\end{array}$ \\
\hline $\begin{array}{l}{[\ldots] \text { entouré par cette }} \\
\text { pourriture glorieuse et } \\
\text { infatuée d'elle-même. } \\
\text { (p. 141) }\end{array}$ & $\begin{array}{l}{[\ldots] \text { surrounded as he }} \\
\text { was by glorious, } \\
\text { self-infatuated } \\
\text { corruption. (p. 108) }\end{array}$ & $\begin{array}{l}{[\ldots] \text { surrounded as he }} \\
\text { was by glorious, } \\
\text { self-infatuated } \\
\text { corruption. (p. 96) }\end{array}$ & $\begin{array}{l}\text { Glissement de sens et } \\
\text { perte de force } \\
\text { péjorative dans les } \\
\text { deux cas }\end{array}$ \\
\hline $\begin{array}{l}{[\ldots] \text { enfin il était sorti de }} \\
\text { cette géhenne. (p. 147) }\end{array}$ & $\begin{array}{l}\text { He was finally out of } \\
\text { that hell. (p. 112) }\end{array}$ & $\begin{array}{l}\text { He was finally out of } \\
\text { that hell. (p. 100) }\end{array}$ & $\begin{array}{l}\text { Légère perte de force } \\
\text { expressive dans les } \\
\text { deux cas en raison du } \\
\text { changement de } \\
\text { registre (banalisation } \\
\text { du mot hell). }\end{array}$ \\
\hline $\begin{array}{l}\text { Cette situation } \\
\text { scabreuse dura un long } \\
\text { moment. (p. 175) }\end{array}$ & $\begin{array}{l}\text { This delicate situation } \\
\text { lasted a long time. } \\
\text { (p. 135) }\end{array}$ & $\begin{array}{l}\text { This delicate situation } \\
\text { lasted a long time. } \\
\text { (p. 120) }\end{array}$ & $\begin{array}{l}\text { Perte de force } \\
\text { péjorative dans les } \\
\text { deux cas. }\end{array}$ \\
\hline $\begin{array}{l}\text { Les femmes sont } \\
\text { intéressées. (p. 235) }\end{array}$ & $\begin{array}{l}\text { Women are selfish. } \\
\text { (p. 179) }\end{array}$ & $\begin{array}{l}\text { Women are selfish. } \\
\text { (p. 161) }\end{array}$ & $\begin{array}{l}\text { Glissement de sens } \\
\text { avec perte de charge } \\
\text { négative. }\end{array}$ \\
\hline
\end{tabular}


Contrairement au terme anglais female, qui désigne le genre de façon neutre, le vocable femelle, appliqué à la femme, prend en français une connotation animale généralement négative. Pour traduire femelles, le traducteur et la traductrice-réviseure ont presque toujours choisi women. Que ce choix résulte de la difficulté à trouver un équivalent ou d'une forme de lissage moral, voire des deux, il en résulte une perte quant à l'intention expressive de l'auteur. Quant au mot putain, chargé d'opprobre et qui peut lui aussi servir d'insulte, il est systématiquement traduit par prostitute, terme beaucoup plus neutre, mais toutefois adéquatement traduit par whore dans des situations de dialogue ${ }^{12}$. Dans un cas (Cossery 1955/2011 : 161, 123,111), Waters a remplacé the young prostitute's death par the young prostitute's murder, mais a maintenu prostitute, intervention qui atténue la charge péjorative de la désignation du personnage tout en rappelant qu'il s'agit d'un meurtre.

\subsection{The Jokers: la traduction d'Anna Moschovakis}

L'examen ${ }^{13}$ de The Jokers (Cossery 1964/2010), la traduction d'Anna Moschovakis de La violence et la dérision (1964), en regard du texte original confirme le resserrement de certaines descriptions, lequel visait vraisemblablement une plus grande idiomaticité. Or, on constate, ici également, quant aux aspects sensibles qui nous intéressent, des interventions d'atténuation ainsi qu'une double omission significative.

TABLEAU 2A

Gestes traductifs d'atténuation observés dans la traduction anglaise de La violence et la dérision

\begin{tabular}{|c|c|}
\hline La violence et la dérision ${ }^{14}$ (Cossery 1964/2005) & $\begin{array}{l}\text { The Jokers, traduction d'Anna Moschovakis } \\
\text { (Cossery 1964/2010) }\end{array}$ \\
\hline $\begin{array}{l}\text { Partager avec une femme des jouissances aussi } \\
\text { délicates lui paraissait inconcevable } \\
\text { Ces femelles étaient absolument imperméables à } \\
\text { cette sorte d'humour [...]. (p. 178) }\end{array}$ & $\begin{array}{l}\text { Inconceivable to share such delicate pleasures } \\
\text { with a woman! } \\
\text { Women were completely impervious to this kind } \\
\text { of worldly humour [...]. (p. 7) }\end{array}$ \\
\hline $\begin{array}{l}\text { La rue s'animait avec les promeneurs du soir [...]. } \\
\text { Mais aucun des consommateurs attablés à la } \\
\text { terrasse du Globe ne faisait attention à cette } \\
\text { morne procession. } \\
\text { Ce n'était pas pour reluquer cette piètre humanité } \\
\text { qu'ils restaient là; tous attendaient avec une } \\
\text { patience émue le passage d'une femelle aux } \\
\text { formes luxuriantes capable de les troubler } \\
\text { charnellement. (p. 221) }\end{array}$ & $\begin{array}{l}\text { The street was packed with evening strollers [...]. } \\
\text { But none of the drinkers at the Globe paid any } \\
\text { attention to this mundane procession. They were } \\
\text { not there to look at humanity in all its mediocrity, } \\
\text { they were waiting } \\
\text { luxuriant curvaceous woman to show up and } \\
\text { arouse their desire. (p. } 44 \text { ) }\end{array}$ \\
\hline $\begin{array}{l}\text { Elle passa - fugitive vision de stupre - emportant } \\
\text { dans le sillage de sa croupe des passions sans } \\
\text { nombre. (p.224) }\end{array}$ & $\begin{array}{l}\text { Then, like a fleeting vision of debauchery, she was } \\
\text { gone, leaving innumerable passions stranded in } \\
\text { her wake. (p. 46) }\end{array}$ \\
\hline $\begin{array}{l}\text { - Les femmes, dit Heykal. Est-ce qu'elles ne } \\
\text { t'amusent pas suffisamment? (p. 224) }\end{array}$ & $\begin{array}{l}\text { - Women, Heykal said. Aren't they enough for } \\
\text { you? (p. 47) }\end{array}$ \\
\hline
\end{tabular}

Deux éléments exprimant la sensualité du regard des clients du café sur les passantes, soit la patience émue et (le sillage) de sa croupe sont supprimés. La formulation Aren't they enough for you? [Ne te suffisent-elles pas ?] élimine la vision de la femme en tant que jouet du désir des hommes. 
TABLEAU 2B

Gestes traductifs d'atténuation observés dans la traduction anglaise de La violence et la dérision (suite)

\begin{tabular}{|l|l|}
\hline \multicolumn{1}{|c|}{ La violence et la dérision (Cossery 1964/2005) } & \multicolumn{1}{c|}{$\begin{array}{c}\text { The Jokers, traduction d'Anna Moschovakis } \\
\text { (Cossery 1964/2010) }\end{array}$} \\
\hline $\begin{array}{l}\text { Depuis qu'il connaissait Heykal, c'est-à-dire } \\
\text { depuis que la vie lui était apparue sous l'aspect } \\
\text { d'une comédie pleine de fureurs dérisoires, } \\
\begin{array}{l}\text { Karim avait abdiqué toute dignité dans ses } \\
\text { rapports avec la tourbe des gens qui détenaient } \\
\text { une parcelle de pouvoir. (p. 188) }\end{array}\end{array}$ & $\begin{array}{l}\text { Since he's known Heykal - that is, since he'd } \\
\text { come to appreciate the comic side of life, with its } \\
\text { many ridiculous passions - Karim had renounced } \\
\text { all dignity in his dealings with ------------- } \\
\text { people who possessed even a modicum of power. } \\
\text { (p. 15) }\end{array}$ \\
\hline $\begin{array}{l}\text { Ce jeune homme était-il sincère? [...] Jamais l'un } \\
\text { de ces révolutionnaires orgueilleux et obstinés, } \\
\text { imbus de leur mission de haine et de mort, } \\
\text { n'aurait employé un tel langage, ni surtout versé } \\
\text { des larmes. (p. 193) }\end{array}$ & $\begin{array}{l}\text { Could the young man be in earnest? [...] These } \\
\text { proud, stubborn revolutionaries, } \\
--------------------------------- \\
\text { would never talk like this, let alone break down } \\
\text { and cry. (p. 19) }\end{array}$ \\
\hline
\end{tabular}

D’un côté, la traductrice supprime un terme, tourbe, qui désigne de façon très péjorative les puissants de la société. De l'autre, elle omet un segment de phrase significatif décrivant l'état d'esprit des révolutionnaires, imbus de leur mission de haine et de mort. Or, ces deux éléments désignaient un aspect crucial de la vision de Cossery: la haine des puissants et le rejet de la révolution classique qui anime ses farceurs de La violence et la dérision.

Elle évite également d'utiliser le f word qui aurait pourtant été l'équivalent culturel dans le contexte:

\section{1. Écoute, Taher! Après tout, je n'ai pas baisé ta sour!}

(Cossery 1964/2005: 297)

Listen, Taher! It's not like I slept with your sister! (p. 297)

(Cossery 1964/2010: 109)

Ce n'est pas que Moschovakis ne soit pas capable d'exprimer la vulgarité de l'original par sa traduction, comme le montrent ces deux exemples:

\section{TABleau $2 \mathrm{C}$}

Gestes traductifs d'atténuation observés dans la traduction anglaise de La violence et la dérision (suite)

\begin{tabular}{|l|l|}
\hline La violence et la dérision (Cossery 1964/2005) & $\begin{array}{l}\text { The Jokers, traduction d'Anna Moschovakis } \\
\text { (Cossery 1964/2010) }\end{array}$ \\
\hline $\begin{array}{l}{[\ldots] \text { resta un moment songeur, à considérer la }} \\
\text { corniche avec une lueur malicieuse dans les yeux. } \\
\begin{array}{l}\text { Il réprimait une forte envie de pisser sur la voie } \\
\text { stratégique. (p. 195) }\end{array}\end{array}$ & $\begin{array}{l}\text { [...] he paused briefly, contemplating the strategic } \\
\text { route with a malicious glint in his eye. He felt a } \\
\text { strong desire to piss on it. (p. 21) }\end{array}$ \\
\hline $\begin{array}{l}\text { Ces mirobolantes croupes }[\ldots] \text { ce festin de } \\
\text { croupes nostalgiques }[\ldots] . \text { La plupart des } \\
\text { propriétaires de ces croupes étaient assez laides } \\
\text { pour faire peur à un assassin sadique. (p. 288) }\end{array}$ & $\begin{array}{l}\text { [...] these amazing asses }[\ldots] \text { this procession of } \\
\text { wistful asses }[\ldots] \text { The owners of the asses were, } \\
\text { for the most part, so ugly then even a sex maniac } \\
\text { would run screaming. (p. 101) }\end{array}$ \\
\hline
\end{tabular}

Bien que la métaphore sensuelle du festin de croupes soit évacuée au profit de l'image beaucoup plus neutre d'une procession, ce dernier exemple présente une amplification (de faire peur à run screaming) et l'ajout d'une connotation sexuelle 
(d'assassin sadique à sex maniac), au lieu de l'atténuation que l'on voit ailleurs. Pourquoi de telles disparités?

Pour tenter de cerner les motifs des traductrices, nous interrogerons la différence entre la perspective postcoloniale fortement présente dans le monde anglo-saxon et la perspective davantage centrée sur l'individu du monde littéraire français dont témoigne la génétique littéraire. Nourries par des paradigmes politico-culturels différents, ces approches créent des attentes différentes à l'égard de la réception de certaines œuvres. Nous recourrons ensuite au concept de tension entre adéquation et acceptabilité de Gideon Toury (1995/2004).

\section{Approche individuelle française, approche postcoloniale américaine}

\subsection{L'effet de la perspective postcoloniale: une recherche davantage théorique et distanciée de l'individu}

La théorie postcoloniale, développée à partir des années 1960 essentiellement dans le monde anglo-saxon, a été appliquée d'abord à la littérature, notamment par Edward Said dans le célèbre Orientalism (1978), par Homi Bhabha dans The Location of Culture (1994) et par Gayatri Spivak dans Can the Subaltern Speak? (1988). Spivak s'intéresse également aux rapports de pouvoir qui se jouent dans la traduction et prône un véritable échange égal, entre cultures. D’autres auteurs ont appliqué à la traductologie la théorie postcoloniale, notamment Bassnett et Trivedi (1999), Tymoczko (1999) et Robinson (1993). En réexaminant l'acte interculturel qu'est la traduction à travers le prisme des relations de pouvoir, le «tournant postcolonial» oblige à le reconsidérer non seulement dans la traduction contemporaine, mais aussi dans l'analyse de traductions d'époques antérieures et dans les retraductions. Ce mouvement, en conjonction avec le «tournant culturel», a notamment nourri Venuti (1986, 1993), parmi les premiers à dénoncer l'hégémonie de la langue anglaise et la «domestication» des textes issus de cultures dominées effectuée par le «traducteur invisible», notion largement reprise dans la discipline.

Plusieurs critiques littéraires anglo-saxonnes de l'œuvre de Cossery révèlent l'influence de la perspective postcoloniale et de son prisme politique. À titre d'exemple, une critique issue de l'Université Oxford déplore son absence dans l'ouvrage Entre Nil et sable (Kober, Fenoglio et al. 1999) soulignant que Gazio ${ }^{15}$ (1999) est le seul parmi ses auteurs à interroger l'identité et la relation à la langue française de Cossery dans une optique sociopolitique:

The volume is clearly directed at a non-specialist, French audience, however, and important questions and problems such as the role of the French language and its ambiguous status in Egypt and what it meant to be "Egyptian" or "Egyptianized" in this period are largely glossed over. The essays do not rigorously challenge the problematic underlying assumption shared by many - that the use of the French language in literature in Egypt is and was unquestionably positive. Pierre Gazio's interesting piece on Albert Cossery is a notable exception to this in that he addresses questions about language, identity and power relations. (Hartman 2001:272)

L'approche génétique d'analyse littéraire, apparue en France dans les années 1970 et qui s'intéresse au processus de création de l'œuvre, est plutôt centrée sur l'individu: 
La génétique des textes repose sur l'hypothèse et l'observation que toute œuvre est le résultat d'un processus de production qui, comme tel, est inscrit dans une temporalité. À partir de l'élucidation des traces graphiques laissées par l'écrivain dans le manuscrit, la génétique se donne pour objectif de reconstituer les différentes étapes du processus d'écriture en les ordonnant dans le temps. Ce travail de déchiffrement, d'analyse et de mise en ordre est un préalable à l'interprétation de la genèse de l'œuvre. (Ganascia, Fengolio et al. 2004: par. 2)

Le professeur Jean Jonassaint, issu du champ francophone d'études littéraires et partisan de l'approche génétique, reproche à Corcoran (2007) de présenter la littérature francophone exclusivement sous une perspective postcoloniale:

[Corcoran] has deliberately inscribed his work in the framework of postcolonial studies. This explicit ideological stand constitutes a bias, which pushes Corcoran to make some questionable choices just to support his thesis of "Francophone literatures as postcolonial literatures". (Jonassaint 2010: 66-67)

Ce cadre analytique semble à Jonassaint réducteur, notamment dans le cas de Cossery:

Should Cossery be considered an Egyptian author, a French writer of Egyptian descent, or an Egyptian by birth, writing, and publishing in French in France? Here, the issue of the post-nationality of contemporary literature, especially the so-called Francophone literatures, and the transnationality of a significant number of writers should be raised. But Corcoran ignores those questions, even though for the most part, the best-known Francophone authors are transnational writers. (Jonassaint 2010: 66-67)

Les chercheurs français ne rejettent pas en bloc la perspective postcoloniale. Jacquemond (2000: 8) a reconnu «la révolution opérée dans les deux dernières décennies par les translation studies et les postcolonial studies». Combe (2011) déplore l'arrivée tardive des études postcoloniales en France (Bhabha et Spivak n'ont été traduits en français qu'en 2007 et 2009 respectivement, soit avec une vingtaine d'années de retard). Combe se montre également critique d'une certaine vision française des littératures francophones:

[L]a situation des études littéraires francophones dans le champ universitaire français n'est sans doute pas pour rien dans l'oubli de la théorie postcoloniale. Si celle-ci est méconnue, voire ignorée, c'est d'abord parce que les littératures dites «francophones» [...] sont jugées «périphériques», voire «mineures» au regard de la littérature «française». (Combe 2011: 18)

Toutefois, en défense à la posture individualisante française, Combe déplore le point de vue "généraliste» (voir ci-dessous) de la perspective postcoloniale:

[L]e point de vue généraliste adopté par la théorie postcoloniale procède d'une lecture insuffisamment contextualisée des œuvres et, inversement, il a pour effet d'abstraire ces mêmes œuvres de leur contexte, essentiel pour comprendre leur genèse, par exemple. [...] Parmi les explications possibles à une «exception française» aux études postcoloniales, il y a donc peut-être le souci du texte dans son historicité et sa matérialité [...] qui produit un net clivage entre les approches critiques «continentales» et anglo-saxonnes. [...] Certes, les critiques anglo-saxons se réfèrent fréquemment au «texte postcolonial». [...] Mais ils donnent avant tout des connotations sociopolitiques au mot [...]. (Combe 2011: 20-21; nous soulignons). 
L'application d'une approche génétique à la traduction est relativement récente, comme le souligne Montini (2016: i) : «À partir de la deuxième décennie de ce millénaire, la génétique textuelle et la traduction se sont frayé un chemin commun à l'intérieur des sciences humaines. Et il était temps [...].» Grutman (2016: 120) propose «la poursuite d'un dialogue entre la génétique des textes et l'autotraduction».

\subsection{Deux exemples anglo-saxons de décontextualisation de l'œuvre de Cossery}

Le choix résolu de la perspective postcoloniale ressort particulièrement dans Botman (1988), publié dans une revue de sociologie. Le résumé annonce avec justesse:

[...] Cossery's personal development as an Egyptian Greek Catholic intellectual whose education and cultural inclination predisposed him to write in French. His life is of particular interest because it mirrors some typical dilemmas faced by minority intellectuals during the transformation of a developing nation from foreign rule to national independence. (Botman 1988: 224)

Botman précise ensuite:

It is the dilemma between personal identification with the masses and cultural estrangement from the populace which deeply marks Cossery's fiction and makes his work worthy of special inquiry. (Botman 1988: 225)

On s'étonnera que l'auteure assimile de façon erronée Cossery à la communauté des Grecs orthodoxes d'Égypte en raison de son appartenance à l'Église «grecquecatholique» et généralise à son cas certaines informations concernant cette communauté, bien distincte de celle des Syro-Libanais (les «Shawam») ${ }^{16}$ dont la famille Cossery était issue. Botman affirme par ailleurs de façon erronée que Cossery «vivait et pensait en français» :

In an important sense, Albert Cossery was an internal exile in Egypt, mainly because he wrote, thought, and lived in a language which differed from the majority of the population. Cossery's use of French, itself an effect of imperialism, marked him off from the vast majority of Egyptians. (Botman 1988: 229; nous soulignons)

Or, on l'a vu, Cossery disait «penser en arabe» (Cossery, dans Cossery et Mitrani 1995: 90). Durant ses années en Égypte, il s’immergeait dans la population pauvre du Vieux-Caire, où il entretenait des relations qui sont au fondement de son inspiration $^{17}$. Il a également affirmé que ses parents ne parlaient pas le français. Par ailleurs, bien qu'elle utilise les guillemets pour le terme language of the "colonizer", l'auteure affirme que le choix du français comme langue d'écriture confère à l'arabe un statut «inférieur» (Botman 1988: 227).

Botman (1988: 231) insiste sur le «pessimisme» et le «nihilisme» qui prévalent selon elle dans l'œuvre de Cossery, sans sembler prendre la mesure de la notion de dérision comme jouissance de la vie pourtant centrale à son œuvre. Certes, en 1988, ni La violence et la dérision (1964) ni Un complot de saltimbanques (1975) n'avaient encore été traduits en anglais, et Cossery n’avait pas encore commencé à donner d'entrevues révélatrices de sa vision du monde. Ces erreurs factuelles constituent toutefois un exemple du manque de contextualisation individuelle de l'analyse d'une œuvre au profit d'une perspective se voulant essentiellement postcoloniale, telle que l'évoque Combe. 
Un ouvrage plus récent adopte une attitude similaire. Parris (2009: s.p. $)^{18}$ souligne lui aussi «l'extraordinaire cohérence de la pensée de Cossery» et tente de démontrer que les indices de l'«égyptianité» (Parris 2009: 135) de l'œuvre cossérienne sont faibles ${ }^{19}$ alors même qu'il a annoncé ne pas être " un expert de la littérature égyptienne et encore moins un arabisant» (Parris 2009: s.p.). Dans son étude systématique et approfondie - toutefois dénuée de références théoriques -, Parris souligne la contradiction entre une affirmation de Cossery - "Mais les femmes occupent la plus grande, la seule place!» (Cossery, dans Cossery et Mitrani 1995: 33) - et ce qu'il observe dans les œuvres, concluant à juste titre que les femmes occupent la portion congrue et se révèlent souvent des personnages peu intelligents, parfois encombrants, seules les fillettes et les adolescentes trouvant grâce aux yeux de l'écrivain.

Parris écrit, à propos de la description d'un quartier dans Les hommes oubliés de Dieu, où «les hommes battaient leurs femmes» et ainsi «régnaient d'une manière inéluctable» (Cossery 1941 : 214):

Même en admettant qu'un auteur ne soit pas responsable de la réalité sociale qu'il est amené à présenter, ce n'est guère [...] la plume d'un féministe convaincu. D’ailleurs, aucune critique de ce point de vue n'est formulée, et les femmes ne prennent aucune revanche. (Parris 2009: 62)

Ce point de vue s'apparente davantage aux approches féministe ou postcoloniale qu'à celle de la génétique littéraire. Enfin, dans sa conclusion, Parris (2009: 153) s'interroge: "L'univers de Cossery est-il absurde? En tout cas, il est loin d'avoir la cohérence de l'univers chrétien [sic]. Il comporte bien des choses qu'il ne faut pas chercher à comprendre».

Ces deux commentaires sur l'œuvre de Cossery (Botman 1988; Parris 2009) illustrent la décontextualisation qu'évoque Combe (2011).

À l'inverse, Espinose amène le lecteur à découvrir la perspective "philosophique» (Espinose 2009: 11) de l'écrivain. Selon lui, les femmes, chez Cossery, ont comme «ciment» de leur ressemblance «l'absence d'intelligence» et «ne peu[ven]t évoluer que dans l'imposture» (Espinose 2009: 69-70). Cependant, l'auteur mentionne les portraits cossériens de très jeunes filles sous une lumière positive, et ajoute:

Il est vrai qu'avec les romans de Cossery, nous sommes transportés, et il ne faut pas l'oublier - surtout lorsqu'il s'agit d'évoquer la condition féminine - dans le monde oriental. L'approche de la femme telle que nous l'évoquons ici doit être perçue dans un environnement social, économique, historique, politique, et même religieux [...] spécifiques. (Espinose 2009: 68)

L'auteur français a donc, lui, effectué une contextualisation explicite et implicite résultant en une perspective nettement différente de celle que communiquent les auteurs anglo-saxons. Le fait que cette contextualisation culturelle constitue une reconnaissance de la culture de l'Autre, valeur faisant également partie du discours postcolonial, montre que les approches centrées sur l'individu et l'approche postcoloniale peuvent théoriquement se chevaucher. 


\section{Sources péritextuelles et épitextuelles d'information sur le positionnement des traductrices et des éditeurs}

Philippe Lane définit ainsi le péritexte et l'épitexte:

Le péritexte se trouve donc «autour du texte» et l'épitexte «autour du livre». Le premier désigne «les genres discursifs qui entourent le texte dans l'espace du même volume: le péritexte éditorial (collections, couverture, matérialité du livre), le nom d'auteur, les titres, le prière d'insérer, les dédicaces, les épigraphes, les préfaces, les intertitres et les notes.» Le second désigne "les productions qui entourent le livre et se situent à l'extérieur du livre: l'épitexte public (épitexte éditorial, interviews, entretiens), l'épitexte privé (correspondance, journaux intimes).» (Lane 1992:18)

\subsection{The Jokers: Une préface révélatrice des normes de la réception}

Un élément péritextuel révèle en quoi l'œuvre de Cossery contient matière à choquer les valeurs de son lectorat américain. La traduction anglaise de La violence et la dérision fait l'objet d'une préface de James Buchan ${ }^{20}$, ancien envoyé spécial au MoyenOrient et collaborateur du Guardian, qui a publié en Days of God: The Revolution in Iran and its Consequences (2012). Son autorité en matière «moyen-orientale» encourage l'adhésion du lectorat à son point de vue. Sa préface, par ailleurs bien documentée, comporte de forts jugements de valeur sur Cossery lui-même et sur ses personnages en ce qui concerne leur vision du peuple égyptien, des femmes et du monde arabe en général:

Cossery [...] like Baudelaire, has no compassion nor sympathy for the poor [and] is fascinated by the division of labor in the very pits of society. [...] Cossery's heroes do not seek virtue [...] but [...] only a natural and malodorous contentment. [Cossery's] marriage was a failure; though whether this was a cause or an effect of his contempt for women, or both, I cannot tell. [...] Females shed all interest at puberty [...]. [...] There is not the slightest interest as to why the poor of Egypt are poor [...] to nobody's surprise but Cossery's, the Arab world has chosen not derision, but violence. (Buchan 2010: vii-x; nous soulignons)

Outre le fait que la préface de Buchan contient certaines assomptions non vérifiables, ces jugements négatifs créent une distance entre le préfacier et l'œuvre, d'une part, et entre le lecteur et l'œuvre, d'autre part. Le préfacier donne l'impression d'avertir un lectorat qu'il voit comme étant politisé, informé, ayant des préoccupations d'ordre politique et humanitaire. Par la publication de cette préface extrêmement critique, l'éditeur signale clairement qu'il ne souscrit pas aux valeurs qu'il croit véhiculées par le texte.

\subsection{Positionnement des traductrices: indications fournies par l'épitexte}

L'épitexte dont nous disposons révèle toutefois, chez les deux traductrices américaines, un attachement envers l'auteur et ses personnages et un grand désir de fidélité à leur voix, en dépit d'obstacles de nature stylistique. 


\subsection{1. Épitexte entourant la traduction d'Alyson Waters}

Alyson Waters, professeure de traduction littéraire à Yale, reçoit plusieurs prix pour ses traductions d'auteurs francophones. L'éditeur New Directions lui a confié la traduction d'Un complot de saltimbanques, publié en 2010 sous le titre A Splendid Conspiracy (Cossery 1975/2010). Sa traduction de Les couleurs de l'infamie paraîtra en 2011 sous le titre The Colors of Infamy (Cossery 1999/2011), chez le même éditeur. Dans la foulée, une réédition de la traduction de Mendiants et orgueilleux (1955) effectuée par Thomas W. Cushing (Cossery 1955/1981) a fait l'objet d'une révision d'Alyson Waters (effectuée après le Printemps égyptien de janvier 2011), qui en signe la longue préface: «Proud Beggars is an excellent introduction to the work of a writer who is greatly admired in France and, in the aftermath of the Arab Spring, is garnering interest around the world»(Waters 2010: vii). La traductrice analyse les personnages et l'œuvre: "The seven novels [...] constitute a tightly unified œuvre, a sort of Egyptian comédie humaine ${ }^{21}$. Cossery's vision of the world was based on abhorrence of abusive power and wealth» (Waters 2010: xii). Alyson Waters, qui décrit la vision cossérienne de la même manière que les critiques français, connaît de toute évidence très bien son œuvre. En entrevue avec Esposito (18 octobre 2011), elle souligne que Cossery, dans ses dialogues,

[...] has a lot of fun with what are supposed to feel like direct translations from the Arabic, with phrases such as "By Allah!" and "Peace be with you," and "Effendi" and "Bey" appearing now and again for "local color." It was an interesting challenge from a translation point of view to keep that distinction between narrative voice and dialogue, without making the dialogues too stilted or odd in English; but they are stilted and odd to the French ear, too, and so that had to be retained. (Waters, dans Esposito 18 octobre 2011 : par. 2)

Waters, qui dit regretter ne pas connaître l'arabe, dit avoir fait appel à des personnes connaissant à la fois le français et l'arabe égyptien afin de s'assurer de la justesse de sa traduction des dialogues. Nous avons donc affaire à une traductrice chevronnée qui contextualise l'œuvre et s'applique à communiquer le message de l'auteur. Dans l'introduction qu'elle a rédigée pour sa révision de Proud Beggars (Cossery 1955/2011), elle mentionne que "a revolutionary strain permeates all of Cossery's work» (Waters 2011 : xiii). Dans ce cas, l'éditeur, New York Review Books, s'appuie sur le statut de spécialiste de cette professeure pour présenter l'ouvrage au lecteur.

Ni l'introduction ni l'entrevue d'Alyson Waters ne comportent de jugement négatif sur Cossery ou sur son œuvre, ou de mention de sa misogynie.

\subsection{2 Épitexte entourant la traduction d'Anna Moschovakis}

La violence et la dérision, publié en 1964, mais traduit en anglais pour la première fois en 2010 par Anna Moschovakis, sous le titre The Jokers, est emblématique de la vision de Cossery. En effet, l'intrigue oppose nettement les tenants de la révolution et ceux de la dérision (les jokers Karim, Heykal et Urfy). Alors que le groupe de plaisantins s'employait à ridiculiser un infâme gouverneur au moyen d'affiches outrageusement flatteuses et donc redoutablement comiques, le triste et peu imaginatif révolutionnaire Taher réussit à l'assassiner, perpétuant le sinistre cycle de la comédie du pouvoir. 
L'entrevue effectuée par Esposito (21 mars 2011) auprès de la traductrice à propos de The Jokers ne présente elle non plus aucune critique négative de la vision cossérienne. Anna Moschovakis, poétesse, traductrice et réviseure, a traduit plusieurs auteurs français; également enseignante universitaire, elle a reçu plusieurs prix ${ }^{22}$. Dans l'entrevue, elle souligne avoir ressenti, à des degrés divers, de la sympathie envers tous les personnages, sur lesquels elle se garde d'émettre des jugements de valeur. Elle précise toutefois s'être sentie plus proche de celui d'Urfy, qui dirige bénévolement une école de quartier. Comparé au gouverneur grotesque et honni, au révolutionnaire enragé ou aux plaisantins délinquants et cyniques, Urfy est le seul personnage dont les comportements sont régis par des valeurs morales.

Par ailleurs - comme Alyson Waters -, Anna Moschovakis explique que le plus grand défi de traduction aura été pour elle l'abondance d'adjectifs et les formulations descriptives de Cossery; elle affirme avoir utilisé une stratégie de réduction pour assurer l'idiomaticité dans la langue cible. La traductrice manifeste un intérêt pour le thème du roman et les liens historico-culturels qui peuvent être faits avec le Printemps égyptien de 2011 (survenu après la publication de la traduction, mais avant l'entrevue).

\section{Positionnement des traductrices : conflit de valeurs entre adéquation de la traduction et acceptabilité de l'auteur}

\subsection{La censure en traduction}

Bien qu'il soit délicat d'évoquer ici des cas de censure, certaines des interventions observées pourraient tout de même s'y apparenter.

La censure en traduction suscite un intérêt croissant de la discipline traductologique (entre autres Ní Chuilleanáin, Ó Cuilleanáin, et al. 2009; Merkle 2002; Billiani 2007; Ballard 2011). Si la majeure partie de ces textes portent sur une censure exercée dans le cadre de régimes répressifs (Allemagne nazie, Italie fasciste, Espagne de Franco), plusieurs examinent la censure exercée par l'agent traduisant en raison d'idéologies dominantes dans leur société, laquelle vise l'acceptabilité de l'œuvre. Notamment, Tymoczko (2009) s'appuie sur la théorie gramscienne de l'hégémonie, qui expliquerait le fait que des traducteurs intègrent les valeurs et les intérêts dominants pour veiller à leurs propres intérêts, ou encore par conviction que leur geste favorisera la réception de l'œuvre au sein du groupe dominant. Par ailleurs, plusieurs auteurs mentionnent la souscription aux normes telle qu'elle est définie par Gideon Toury.

\subsection{La tension entre adéquation et acceptabilité sociale d'une traduction selon Toury}

Selon Toury (1995/2004), la traduction est une activité culturelle, et le traducteur joue un rôle social qui est donc soumis à un ensemble de normes. Les normes sociales, qui fluctuent entre règles absolues et caractéristiques idiosyncratiques, sont acquises par l'individu au cours de sa socialisation. Tyulenev (2014) mentionne d'ailleurs l'importance de la socialisation individuelle et professionnelle du traducteur, laquelle, selon lui, demeure évolutive au cours du développement de sa pratique. Toury affirme 
que la traduction est soumise à une tension bipolaire entre les normes de la culture source et celles de la culture cible. Le pôle de l'adéquation correspond à la plus grande adhésion possible aux premières, et le pôle de l'acceptabilité, à la plus grande adhésion possible aux secondes. Cette tension s'applique à toute culture et à tout type de traduction. On ne peut observer les normes elles-mêmes; on en opère une reconstitution (reconstruction) dans les champs textuel et extratextuel (Toury 1995/2004: 213).

Selon Toury, l'obtention de l'équivalence repose de façon absolue sur le statut de la traduction - activité et produit - au sein de la culture cible. Enfin, il signale que plusieurs "générations de normes» peuvent coexister, justement en raison de leur caractère socialement déterminé et fluctuant, et insiste sur leur complexité en tant qu'objet d'étude (Toury 1995/2004: 205).

Toury distingue les normes «préliminaires» (preliminary norms), qui concernent des gestes faits en amont de l'acte traductif (politiques, modalités contextuelles), et les «normes opératoires» (operational norms), qui entourent le processus de traduction lui-même. Ainsi, la préface fortement critique de James Buchan à The Jokers mentionnée plus haut, en avertissant le lecteur de ce qui risque de lui déplaire, voire l'indigner, permettrait de «reconstituer» certaines normes «préliminaires » régissant l'acceptabilité de l'œuvre.

Enfin, selon lui, et cela nous intéresse particulièrement dans les cas étudiés, certaines interventions à un niveau "micro» peuvent s'éloigner de la stratégie de traduction générale adoptée:

[...] adherence to source norms determines a translation's adequacy as compared to the source text, subscription to norms originating in the target culture determines its acceptability. [...] Even if no clear macro-level tendency can be shown, any micro-level decision can still be accounted for in terms of adequacy vs. acceptability. On the other hand, in cases where an overall choice has been made, it is not necessary that every single lower-level decision be made in full accord with it. (Toury 1995/2004: 201; nos italiques)

Ainsi, le fait que certaines interventions visent une plus grande acceptabilité n'empêche pas que la stratégie générale adoptée corresponde bien à la recherche de l'adéquation.

\subsection{La complexité du positionnement des traductrices}

Les commentaires extrêmement positifs des deux traductrices sur l'auteur et sur ses personnages, sur le désir de rendre sa langue, de faire entendre sa voix unique nous semblent typiques de la posture bermanienne consistant à accueillir l'autre « en tant qu'autre» (Berman 1985: 88). Or, il s'agit ici de faire entendre, en quelque sorte, au moins deux cultures, la culture égyptienne et la culture française. Et de quelle culture égyptienne, de quelle culture française parlons-nous? Il s'agit en fait de la rencontre littéraire qui s'est produite dans l'effervescence de l'après-guerre, à la fois au Caire et à Paris. En outre, saisir la dimension politico-satirique de l'expérience égyptienne de Cossery sans perdre de vue sa part intensément humaine exige de la part de ses traducteurs une connaissance profonde de l'auteur, de son parcours et de son héritage littéraire. Cette connaissance - on pourrait dire cette connivence - qui filtre des entrevues données par les deux traductrices contraste avec la perplexité de Parris (2009: 153) devant un monde où, selon lui, «il y a des choses qu'il ne faut pas chercher à comprendre». 
La bipolarité décrite par Toury est bien présente. Du côté de l'adéquation de la traduction, il y a la mission de traduire le monde de Cossery de manière lisible, cohérente. Quant à l'acceptabilité sociale, elle cible ici les lecteurs de Cossery aux États-Unis. Dans les années 1950, il s'agissait d'un lectorat s'intéressant au mouvement littéraire parisien d'après-guerre. Le lectorat des années 2000 a connu le tournant postcolonial, l'avènement du féminisme et du postcolonialisme. Informé, politisé, ce groupe social est particulièrement sensible au traitement des femmes et des groupes ethniques "non blancs». Spivak (1988) illustre bien la complexité du positionnement à l'égard de l'autre dans un contexte postcolonial.

Féminisme, respect de l'autre, quelle que soit son origine ethnique, et respect des classes sociales opprimées appartiennent à la même famille de valeurs. À partir du moment où ces valeurs sont devenues dominantes chez le lectorat potentiel de Cossery, nous supposons que ses traducteurs se retrouvent dans ce que l'on pourrait appeler un conflit de loyautés idéologiques. En sus de la notion de double contrainte qui traverse sous diverses formes et appellations l'ensemble de la traductologie, il y a une contrainte multiple du côté même du texte source: s'agit-il de littérature française, égyptienne, hybride? Jusqu'où être fidèle à l'auteur en le rendant lisible dans les détails qui le rendent unique, sans pour autant qu'il soit «inacceptable» pour les lecteurs?

Ainsi, le traducteur et les deux traductrices auront, de façon consciente ou inconsciente - cette notion elle-même étant sujette à débat -, négocié un équilibre entre la fidélité à la voix de l'auteur et celle aux valeurs du lectorat au moyen d'opérations de lissage (une véritable censure pouvant se discuter pour deux cas). Par ailleurs, comment expliquer les interventions à géométrie variable d'Anna Moschovakis dans The Jokers? Dans ce dernier cas, nous avançons prudemment que la traductrice pourrait s'identifier à la voix de l'auteur-narrateur: en effet, les occurrences de lissage et les suppressions sont effectuées dans la narration, tandis que les quelques amplifications de la vulgarité portent sur les dialogues. Si Cossery a maintes fois affirmé qu'il s'exprime par la bouche de ses personnages, les déclarations du narrateur ont un poids moral plus prégnant. On pourrait même envisager que les cas d'amplification de la charge vulgaire soient une forme de "compensation» des rognages effectués ailleurs.

\section{Conclusion}

Milner (2011 : 31) fait une distinction entre «penser de manière massive» et «penser en détail». L'importance de ce deuxième pôle en traduction est d'ailleurs soulignée par Cronin (2013: 197). Dans les traductions anglaises de La violence et la dérision et de Mendiants et orgueilleux, ainsi que dans la révision de cette dernière traduction, certains «détails» de passages possédant une charge de vulgarité, de misogynie ou de dureté apparente envers le "peuple» égyptien ont été rabotés, dilués, voire omis. Nous avons tenté d'éclairer ces gestes à la lumière de différences culturelles présentes dans les champs anglo-saxon et français de la critique littéraire, à savoir une plus forte présence de l'approche postcoloniale en terrain anglo-saxon et une vision plus individuelle de l'œuvre et de l'auteur dans la perspective française.

En faisant appel aux notions d'intériorisation des normes et de tension entre adéquation et acceptabilité sociale (Toury 1995/2004), nous avons ensuite tenté de 
comprendre le positionnement des traductrices et les conflits à l'égard des valeurs qui ont pu sous-tendre leurs gestes de lissage, voire une éventuelle (auto)censure. Selon Toury:

Censorship can [also] be activated during the act of translation itself [...], inasmuch as the translator has internalized the norms pertinent to the culture, and uses them as a constant monitoring device. (Toury 1995: 278)

Certes, il ne nous est pas possible de savoir quelle est la part des pressions internes et externes (Merkle 2004 ${ }^{23}$ ), ni si ces gestes traductifs ont été effectués de manière consciente ou inconsciente.

Quoi qu'il en soit, la tension entre fidélité à l'auteur et acceptabilité sociale en traduction littéraire demeure à investiguer, notamment dans un contexte où des pressions liées aux attentes en évolution du lectorat s'exercent de façon croissante dans l'espace public. Ces pressions, qui touchent l'ensemble de la production écrite, posent un problème particulier dans le cas de rééditions, de retraductions ou encore de traductions d'œuvres non contemporaines.

\section{REMERCIEMENTS}

L'auteure souhaite souligner l'aide financière accordée par le Comité paritaire de formation professionnelle et de perfectionnement de l'Université de Montréal et du Syndicat des chargés et chargées de cours de l'Université de Montréal pour la rédaction de cet article.

\section{NOTES}

1. Cossery, Albert (2005): Albert Cossery, petits tableaux de la sagesse orientale. (Entrevue par Aliette ArmeL) Le Magazine littéraire. 447:92-97.

2. Cette information est établie notamment à partir des entrevues publiées et de Fenoglio (1999).

3. Tels Karim (La violence et la dérision) et Karamallah (Les couleurs de l'infamie), qui ont payé d'un passage en prison leurs élans révolutionnaires.

4. Jacquemond donne l'exemple de la traduction d'André Miquel d'un roman de Naguib Mahfouz, qui «guide le lecteur» tout en «exotisant» le texte (Jacquemond 1993: paragraphe 29).

5. Golo (2009): Mendiants et orgueilleux. (Adapté du roman éponyme d'Albert Cossery) Paris: Futuropolis.

6. Leur publication a été subventionnée par le ministère français des Affaires étrangères et le Service culturel de l'ambassade de France aux États-Unis.

7. L'idéologème est ainsi défini par Angenot (1977: 24): «Nous appellerons "idéologème" toute maxime, sous-jacente à un énoncé, dont le sujet circonscrit un champ de pertinence particulier (que ce soit "la valeur morale", "le Juif", "la mission de la France" ou "l'instinct maternel")."

8. En outre, dans La maison de la mort certaine, œuvre de jeunesse de Cossery, c'est un groupe de femmes qui aura le courage d'affronter le veule propriétaire. Et un personnage de femme âgée se démarque, celui de la mère d'Urfy, à qui la folie semble apporter une connaissance pure du monde et qui fascine Heykal, un des héros de La violence et la dérision, qui voit en elle une transcendance à laquelle il aspire.

9. Comme celle de Post (2010), qui affirme à propos de The Jokers: "Cossery [is] one of the most interesting international authors - living or dead - to be published in America this year», ou celle de Orthofer (2010), qui souligne à propos du même roman: «It's also hard not to be enthralled by Cossery's writing [...] in its subtle, playful way [this novel] is as politically subversive as many much louder and more violent works. And it's very good fun.»

10. Notre traduction de "retain a similar lexicon in the three books» et «keep the idiosyncrasies of Cossery's style the same». Puisque les héros masculins, on l'a vu, expriment la voix de Cossery.

11. Le mot putain présent dans la narration en six occurrences (Cossery 1955/2013: 68, 71, 91, 161, 187, 238) est traduit par prostitute par Cushing (Cossery 1955/1981:53, 55, 70, 123, 143, 182) et laissé comme tel par la révision de Waters (Cossery 1955/2011: 45, 47, 61, 111, 129, 173). 
12. Par exemple, Même un cul de putain réserve des surprises (Cossery 1955/2013: 190) est rendu par Even a whore's ass holds surprises par Cushing (Cossery 1955/1981: 145) et conservé tel quel par Waters (Cossery 1955/2011: 131).

13. Les suppressions sont marquées par un espace vide; celles potentiellement liées à une autocensure sont en gris foncé.

14. Dans le tome 2 des Euvres complètes, d'où l'écart entre les numéros des pages.

15. Gazio a par ailleurs rédigé en 1990 une thèse de doctorat sur ce sujet; celle-ci n'est disponible ni en format numérisé ni par le prêt interbibliothèque.

16. Certes, une partie de cette population avait adopté le français dans sa vie quotidienne. Hussam Eldin (2011) décrit cette communauté qui a joué un rôle économique (commerce) et culturel (cercles littéraires francophones) au Caire avant que le mouvement nationaliste (revendiquant en tant que fondements la langue arabe et l'islam) ainsi que les confiscations des biens ayant suivi la révolution nassérienne ne mènent à l'expatriation d'un grand nombre d'entre eux.

17. Il mentionne que les personnages de ses romans ont tous existé, qu'il les a connus (Cossery, dans Cossery et Mitrani 1995: 55, 71, 93).

18. Citation extraite des «Remarques liminaires» en début d'ouvrage.

19. Notamment, Parris (2009) semble regretter à plusieurs reprises que les lieux ne soient pas nommés en laissant entendre que cela affaiblirait l'égyptianité des œuvres.

20. Voir le profil de James Buchan sur le site Web du Guardian, qui rassemble ses diverses recensions d'ouvrages. Consulté le 10 octobre 2016, <http://www.theguardian.com/profile/jamesbuchan>.

21. En français dans le texte.

22. Voir la fiche d'Anna Moschovakis sur le site Web de la Poetry Foundation. Consulté le 12 mai 2016, $<$ https://www.poetryfoundation.org/poets/anna-moschovakis >.

23. Merkle, Denise (2004): External and Internal Pressures on the Translator: Relationship to censorship. International Comparative Literature Association. Consulté le 3 avril 2019, <http://www. ailc-icla.org/2004/Denise\%20Merkle.doc>.

\section{RÉFÉRENCES}

Andrau, Frédéric (2013): Monsieur Albert: Cossery, une vie. Clichy: Éditions de Corlevour. Angenot, Marc (1977): Présupposé, topos, idéologème. Études françaises. 13(1-2):11-34.

Ballard, Michel, dir. (2011): Censure et traduction. Arras: Artois Presses Université.

Bassnett, Susan et Trivedi, Harish, dir. (1999): Postcolonial translation: Theory and practice. Londres/New York: Routledge.

Berman, Antoine (1985): La traduction et la lettre ou l'auberge du lointain. In: Antoine Berman, Gérard Granel, Annick Jaulin et al., dir. Les tours de Babel: essais sur la traduction. Mauvezin: Trans-Europ-Repress, 35-150.

Bнавна, Homi K. (1994): The postcolonial and the postmodern: The question of agency. In: Homi K. Внавна. The Location of Culture. Londres/New York: Routledge, 171-197.

Billiani, Francesca (2007): Modes of Censorship: National Contexts and Diverse Media. Londres/New York: Routledge.

Bitton, Jean-Luc (1996) : Albert Cossery: Salam alhikoum, Salut sur toi! Nuit blanche. (63):6264.

Botman, Selma (1988): Albert Cossery and the men God forgot: Some aspects of the ethnic subcultures of Egyptian society. Immigrants \& Minorities. 7(2):224-237.

Buchan, James (2010): Introduction. In: Albert Cossery. The Jokers. (Traduit du français par Anna Moschovakis) New York: New York Review Books, vii-x.

Buchan, James (2012): Days of God: The Revolution of Iran and its Consequences. Londres: John Murray.

Christin, Rodolphe (2017): Le Désert des ambitions. Avec Albert Cossery. Paris: Éditions L'échappée.

Сомвe, Dominique (2011): «Le texte postcolonial n'existe pas»: Théorie postcoloniale, études francophones et critique génétique. Genesis. 33:15-28.

Corcoran, Patrick (2007): The Cambridge Introduction to Francophone Literature. Cambridge/ New York: Cambridge University Press. 
Cossery, Albert et Mitrani, Michel (1995): Conversations avec Albert Cossery. Paris: Éditions Joëlle Losfeld.

Cronin, Michael (2013) : Mind the Gap: Translation Automation and the Lure of the Universal. TTR. 26(2):193-218.

Espinose, Raymond (2009): Albert Cossery, une éthique de la dérision. Paris: Orizons.

Fenoglio, Irène (1999): L'égyptianité d'Albert Cossery. In: Marc Kober, Irène Fenoglio et Daniel Lançon, dir. Entre Nil et sable: écrivains d'Egypte d'expression française (1920-1960). Paris: Centre national de documentation pédagogique, 127-133.

Fili-Tullon, Touriya (2009): Figures de la subversion dans les littératures francophone et d'expression arabe au Maghreb et au Proche-Orient, des années 1970 à 2000 (R. Boudjedra, A. Cossery, E. A. El Maleh, É. Habibi et P. Smaïl). Thèse de doctorat non publiée. Paris: Université Paris 3.

Ganascia, Jean-Gabriel, Fenoglio, Irène et Lebrave, Jean-Louis (2004) : Manuscrits, genèse et documents numérisés. EDITE: une étude informatisée du travail de l'écrivain. Document numérique. 8(4):91-110.

Gazio, Pierre (1990): L'œuvre romanesque d'Albert Cossery: écrivain d'Égypte francophone. Thèse de doctorat non publiée. Pessac: Université Bordeaux Montaigne.

Gazio, Pierre (1999): Le savoir-vivre égyptien d'Albert Cossery. In: Marc Kober, Irène FenoGLIO et Daniel LANÇON, dir. Entre Nil et sable: écrivains d'Égypte d'expression française (1920-1960). Paris: Centre national de documentation pédagogique, 105-112.

Grutman, Rainier (2016): Manuscrits, traduction et autotraduction. In: Chiara Montini, dir. Traduire. Genèse du choix. Paris: Édition des archives contemporaines, 115-128.

Hartman, Michelle (2001): Compte-rendu de Entre Nil et sable: écrivains d'Égypte d'expression française (1920-1960) dirigé par Marc Kober, Irène Fenoglio et Daniel Lançon [1999, Paris: Centre national de documentation pédagogique]. French Studies. 55(2):272-273.

Henein, Georges (1956) : L'apport d'Albert Cossery. Calligrammes. [?] :17-23.

Hussam Eldin, Raafat Ahmed (2011): From Nahda to exile: a story of the Shawam in Egypt in the early twentieth century. Mémoire de maîtrise non publié. Montréal: McGill University.

JACQUemond, Richard (1993): Traductions croisées Égypte-France: stratégies de traduction et échange culturel inégal. Égypte/Monde arabe. 15-16: 11p. Consulté le 14 janvier 2019, $<$ http://journals.openedition.org/ema/1109>.

JACQUEMOND, Richard (2000): Langues étrangères et traduction dans le champ littéraire égyptien. Alif: Journal of Comparative Poetics. 20:8-38.

Jonassaint, Jean (2010) : Compte-rendu de The Cambridge Introduction to Francophone Literature par Patrick Corcoran [2007, Cambridge/New York: Cambridge University Press]. Symposium. 64(1):66-68.

Khalifa, Ahmed Mahmoud (2012): Les traductions arabes des cuvres des écrivains francophones d'origine égyptienne. Étude sociolinguistique de la réception. Thèse de doctorat non publiée. Le Caire: Université al-Azhar.

Kober, Marc (1999): L'inaction et le cri au miroir. In: Marc Kober, Irène Fenoglio et Daniel LANÇON, dir. Entre Nil et sable: écrivains d'Égypte d'expression française (1920-1960). Paris: Centre national de documentation pédagogique, 113-126.

Kober, Marc (2016): Comment raconter Le Caire? Dynamique littéraire et mutations urbaines. Itinéraires. 2016-1:20p. Consulté le 23 septembre 2017, <https://journals.openedition.org/ itineraires/3254>.

Kober, Marc, Fenoglio, Irène et Lançon, Daniel (1999): Entre Nil et sable: écrivains d'Égypte d'expression française (1920-1960). Paris: Centre national de documentation pédagogique.

Lane, Philippe (1992): La périphérie du texte. Paris: Nathan.

Losfeld, Joëlle (2005) : Avant-propos de l'éditrice. In: Albert Cossery. CEuvres complètes. Vol. II. Paris: Éditions Joëlle Losfeld/Gallimard.

Merkle, Denise, dir. (2002): Censure et traduction dans le monde occidental. TTR. 15(2).

Milner, Jean-Claude (2011): Clartés de tout: de Lacan à Marx, d'Aristote à Mao. Lagrasse: Verdier. 
Montini, Chiara (2016): Traduire. Genèse du choix. Paris: Éditions des archives contemporaines.

Ní Chuilleanáin, Eiléan, Ó Cuilleanáin, Cormac et Parris, David L., dir. (2009): Translation and Censorship: Patterns of Communication and Interference. Dublin: Four Courts Press.

PARris, David L. (2009): Albert Cossery. Montreur d'hommes. Berne: Peter Lang.

Robinson, Douglas (1993): Decolonizing translation. Translation and Literature. 2(2):113-124.

SAID, Edward W. (1978): Orientalism. New York: Pantheon Books.

SpIVAK, Gayatri C. (1988): Can the Subaltern Speak? In: Cary Nelson et Larry GrossBerg, dir. Marxism and the Interpretation of Culture. Champaign: University of Illinois Press, 271-313.

Tyмосzко, Maria (1999): Post-colonial writing and literary translation. In: Susan BAssnetT et Harish TRIVEDI, dir. Postcolonial translation: Theory and practice. Londres/New York: Routledge, 19-40.

Tүмосzко, Maria (2009): Censorship and self-censorship in translation: ethics and ideology, resistance and collusion. In: Eiléan Ní Chuilleanáin, Cormac Ó Cuilleanáin et David L. PARris, dir. Translation and Censorship: Patterns of Communication and Interference. Dublin: Four Courts Press, 24-45.

Tyulenev, Sergey (2014): Translation and society: An introduction. Londres/New York: Routledge.

Toury, Gideon (1995): Descriptive Translation Studies and Beyond. Amsterdam/Philadelphie: John Benjamins.

Toury, Gideon (1995/2004): The nature and role of norms in translation. In: Lawrence VenUTI, dir. The Translation Studies Reader. $2^{\mathrm{e}}$ éd. Londres/New York: Routledge, 205-218.

Venuti, Lawrence (1986): The Translator's Invisibility. Criticism. 28(2):179-212.

Venuti, Lawrence (1993): Translation as cultural politics: Regimes of domestication in English. Textual Practice. 7(2):208-223.

Waters, Alyson (2011): Introduction. In: Albert Cossery. Proud Beggars. (Traduit du français par Thomas W. Cushing et révisé par Alyson Waters) New York: New York Review Books, i-xv.

\section{ANNEXES}

Annexe 1: Corpus

Cossery, Albert (1941): Les hommes oubliés de Dieu. Vol. 1. Le Caire: La Semaine égyptienne. Cossery, Albert (1944): La maison de la mort certaine. Le Caire: Éditions Masses.

Cossery, Albert (1941/1946): Men God Forgot. (Traduit du français par Harold Edwards) Berkeley: Circle Editions.

Cossery, Albert (1948): Les fainéants dans la vallée fertile. Paris: Domat.

Cossery, Albert (1944/1949): The House of a Certain Death. (Traduit du français par Stuart B. KaISer) New York: New Directions Publishing.

Cossery, Albert (1948/1952): The Lazy Ones. (Traduit du français par Stuart B. KAISER) New York: New Directions Publishing.

Cossery, Albert (1955): Mendiants et orgueilleux. Paris: Julliard.

Cossery, Albert (1964): La violence et la dérision. Paris: Julliard.

Cossery, Albert (1975): Un complot de saltimbanques. Paris: Éditions Robert Laffont.

Cossery, Albert (1955/1981): Proud Beggars. (Traduit du français par Thomas W. Cushing) Santa Barbara: Black Sparrow Press.

Cossery, Albert (1984): Une ambition dans le désert. Blanche. Paris: Gallimard.

Cossery, Albert (1999): Les couleurs de l'infamie. Paris: Éditions Joëlle Losfeld.

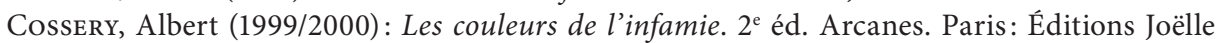
Losfeld.

Cossery, Albert (1964/2005): La violence et la dérision. In: Albert Cossery. CEuvres complètes. Vol. 2. Littérature/Joëlle Losfeld. Paris: Gallimard, 171-337.

Cossery, Albert (1984/2005): Une ambition dans le désert. In: Albert Cossery. Euvres complètes. Vol. 2. Littérature/Joëlle Losfeld. Paris: Gallimard, 339-529. 
Cossery, Albert (2005) : Euvres complètes. Littérature/Joëlle Losfeld. Paris: Gallimard.

Cossery, Albert (1964/2010): The Jokers. (Traduit du français par Anna Moschovakis) New York: New York Review Books.

Cossery, Albert (1975/2010): A Splendid Conspiracy. (Traduit du français par Alyson Waters) New York: New Directions.

Cossery, Albert (1955/2011): Proud Beggars. (Traduit du français par Thomas W. Cushing et révisé par Alyson Waters) New York: New York Review Books.

Cossery, Albert (1999/2011): The Colors of Infamy. (Traduit du français par Alyson Waters) New York: New Directions.

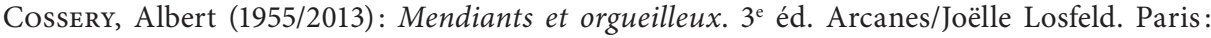
Gallimard.

\section{Annexe 2: Pages Web et articles de presse en ligne portant sur Cossery}

Aïssaoui, Mohammed (27 juin 2008): Albert Cossery: adieu au «Voltaire du Nil». Le Figaro. Consulté le 17 septembre 2017, <http://www.lefigaro.fr/livres/2008/06/23/03005-20080623 ARTFIG00403-albert-cossery-adieu-au-voltaire-du-nil.php>.

Ammoun, Denise (26 juin 2008): Albert Cossery, le «Voltaire du Nil». La Croix. Consulté le 17 septembre 2017, <https://www.la-croix.com/Archives/2008-06-26/Albert-Cossery-leVoltaire-du-Nil-_NP_-2008-06-26-322865>.

Ayad, Christophe (1 $1^{\text {er }}$ décembre 1999): Dernier dandy à Paris. Portrait. Libération. Consulté le 15 mai 2016, <http://www.liberation.fr/portrait/1999/12/01/dernier-dandy-a-paris_292189>.

Esposito, Scott (21 mars 2011): Six Questions for Anna Moschovakis on The Jokers by Albert Cossery. Conversational Reading. Consulté le 15 mai 2019, <https://web.archive.org/ web/20160323175227/http://conversationalreading.com/six-questions-for-anna-moschovakis-on-the-jokers-by-albert-cossery/>.

Esposito, Scott (18 octobre 2011): Alyson Waters on The Colors of Infamy. Center for the arts of translation. Consulté le 20 juin 2019, <https://web.archive.org/web/20161107221054/http:// www.catranslation.org/blogpost/alyson-waters-on-the-colors-of-infamy-by-albert-cossery $>$.

Leroy, Jean-Claude (19 juin 2011): Albert Cossery ou l'efficacité révolutionnaire, itinéraire d'un écrivain égyptien de langue française. Mediapart. Consulté le 10 décembre 2017. <https:// blogs.mediapart.fr/jean-claude-leroy/blog/190611/albert-cossery-ou-lefficacite-revolutionnaire-itineraire-dun-ecrivain-egyptien-de-langue-fran $>$.

McCanne, Michael (5 avril 2012): Revolutionary Laughter. The New Inquiry. Consulté le 22 mars 2019, <https://thenewinquiry.com/revolutionary-laughter/>.

Orthofer, Michael A. (29 juin 2010): The complete review's Review: The Jokers by Albert Cossery. the complete review. Consulté le 10 mai 2016, <http://www.complete-review.com/ reviews/egypt/cossery2.htm>.

Polizotti, Mark (22 mai 2012): Extreme Indolence: On the Fiction of Albert Cossery. The Nation. Consulté le 16 septembre 2017, <http://www.thenation.com/article/extremeindolence-fiction-albert-cossery/>.

Post, Chad W. (28 novembre 2010): Critical review of The Jokers. Three Percent. Consulté le 10 mai 2019, <https://web.archive.org/web/20101202020050/http://www.rochester.edu/ College/translation/threepercent/index.php?id=2964>.

\section{Annexe 3: Films}

Une vie dans la journée d'Albert Cossery (2005): Réalisé par Leys, Sophie. Groupe de recherche et d'essais cinématographiques. France.

Hôtel La Louisiane (2015): Réalisé par La Veaux, Michel. Les Films Outsider. Canada. 